\title{
A practical approach to floodplain mapping for large-scale catastrophe models
}

\author{
I. Carnacina \& A. Jemberie \\ Research and Modeling, AIR Worldwide, USA
}

\begin{abstract}
Catastrophe models often cover large geographic areas spanning multiple countries or, in the case of flood models, entire watersheds. Models must be sufficiently detailed to accurately account for hydrologic variation, which is notably challenging when the modeled region is large. This is particularly true for flood models, which require a highly detailed dataset, usually derived from a digital terrain model (DTM), for reliable floodplain mapping. For one-dimensional (1D) hydraulic models, the floodplain mapping approach tends to yield flat surfaces often resulting in artefacts and inconsistencies near river confluences. Because flood extent is limited by the length of cross-sectional lines along the floodplain, these flat surfaces tend to drop sharply when the simulation reaches a flat delta. The use of a two-dimensional (2D) model avoids these problems, but at a high computational cost, and requires high quality terrain and bathymetry data. This paper presents a new methodology for mapping floodplains using water elevation points along a river network obtained from a 1D hydraulic model and a DTM. The methodology applies kinematic and diffusion wave equations in a simplified manner, using water elevation points as internal boundary conditions. Several parameters control the expansion and smoothing algorithms that generate realistic flood extent maps for different return periods. This methodology is particularly suitable for modeling large domains since it produces accurate results but requires much less computational time than a $2 \mathrm{D}$ model. In addition, because the computation uses several source points per cross section, the flood extent is not limited by the cross-sectional length, making this methodology appropriate for levee breaches and in cases where river banks are not well defined and the crosssectional geometry is derived from a DTM.
\end{abstract}

Keywords: risk assessment, flood, flood mapping, large scale model. 


\section{Introduction}

Flood events in combination with human activity and land use changes threaten both life and property in much of the world. The human and economic losses inflicted by flood events have forced communities and governments to adopt new direct and indirect measures to prevent, assess, and reduce the risk of flooding. Over the last thirty years, a plethora of 1D, 2D and coupled flood models have been developed, most of which are commercially available. Risk maps often require the evaluation of risk for a given non-exceedance probability (often referred to as return period maps), and thus, steady state models are often preferred, again for their computational efficiency [1]. Flood hazard maps are typically produced by governmental agencies, such as FEMA in United States, ZÜRS in Germany, and many others across Europe. In this context, large scale catastrophe risk models are catching the attention of more and more researchers in academia and in industry. These models are used to assess the effect of catastrophic flood on larger areas as opposed to local and detailed studies.

Despite the large number of such models, the need for nationwide medium-tohigh resolution inundation maps has led to the development of fast numerical solutions with reduced computational effort.

In this study, we provide a tool for mapping hydraulic model results from a 1D steady state model using a simplified quasi-physical approach that alternates between the use of kinematic waves and diffusion waves to interpolate water elevations between cross sections. This methodology eliminates the presence of artefacts and drops in flood maps at confluence and reduces the computational effort required in comparison to $2 \mathrm{D}$ models. The same kinematic and diffusion waves approach is used to predict the flow level through a breach, using, as boundary conditions, the solution obtained from 1D numerical model and a volume hydrograph. Finally, the mapping algorithm provides an envelope of the maximum depth for a given return period.

\section{Methodology}

One dimensional steady state models can rapidly assess the intensity and the extent of flooding at specific return periods, provided that the effect of floodplain storage is negligible $[2,3]$ and provided that the accuracy of such models is checked at stream junctions, branches, and lateral inflow [1,4]. One dimensional model results, however, are only available locally at predefined model cross sections. Therefore, 1D model solutions need to be interpolated between cross sections to fill the gaps between them and can also be used in conjunction with 2D models to simulate levee overtopping or flow through levee breaches.

Examples of interpolation of 1D hydraulic model results to create a flood extent map include triangular irregular networks (TIN) generated by HEC-GeoRAS, as well as chained interpolation between cross sections in MIKE-11.

The industry often requires large scale models (often national or continental) at medium-to-high resolutions (specifically, $30 \mathrm{~m}$ to $90 \mathrm{~m}$ ). DTMs and land use datasets provide an essential source of input to generate such large scale models. 
Therefore, model cross sections can be automatically extracted from these datasets. However, given the large spatial coverage of catastrophe models, automatic cross section generation may lead to issues such as:

1) Over large areas, not every cross section can be checked to assess whether it covers the whole floodplain, especially at high return periods (typically, greater than 100 years), thus TIN mapping may create artefacts and sudden drops of water elevation in flat flood plains;

2) For large scale systems, automatically generated cross sections may not cover the entire floodplain and may be too short near stream junctions.

Moreover, the presence of flood defences drastically modifies the dynamics of flow between the main channel and the floodplain. While flood extent maps can be correctly modeled for relatively small flood defences protecting a small portion of urban areas, complex systems of levees such as those protecting the Mississippi or Sacramento rivers create totally disconnected networks, for which the effect of storage is no longer negligible. While 1D models work efficiently inside the leveed areas, water flowing through a breach or overtopping this system of protection needs to be treated separately from the rest of the network.

This methodology uses a quasi-physical raster-based approach to create a water surface between crosssections. The algorithm uses a wave propagation concept that avoids artefacts at the end of cross-section cut lines and river junctions that are yielded by TIN-based interpolation. This methodology is also used to assess the effect of levee breach or overtopping based on the solution obtained from the 1D model.

\subsection{General framework}

Free surface wave propagation along rivers is generally approximated using the de Saint-Venant equation [7, 8]:

$$
\underbrace{\frac{1}{A} \frac{\partial Q}{\partial t}+\frac{1}{A} \frac{\partial\left(Q^{2} / A\right)}{\partial t}+\underbrace{g \frac{\partial y}{\partial x}-\underbrace{g\left(S_{0}-S_{f}\right)}_{\text {Dave }}}_{\text {Kinematic wave }}}_{\text {Diffusion wave }}=0
$$

Dynamic wave

where $Q$ is the discharge trough of a given cross-section or computational node, $A$ is the cross-sectional area, $g$ is the gravitational acceleration, $y$ is the depth of flow, $S_{0}$ is the bottom slope, and $S_{f}$ is the friction slope.

Depending on the terms included to approximate the momentum balance (1), the wave propagation assumes different names: kinematic wave, diffusion wave, and fully dynamic propagation. In the present work (steady state conditions), only the kinematic and diffusion waves are considered.

To interpolate the solution of the 1D model (water elevation $\zeta$ ) from the cross section cut lines to the rest of the domain (DTM), a set of points along the cross section is chosen to serve as source points (subscript $\mathrm{S}$ ).

The algorithm is then divided in two phases: 1) a first expansion phase, in which the solution of the $1 \mathrm{D}$ source cell is propagated to the rest of the domain, 
and 2) a second smoothing phase, in which the water elevations from the expansion phase are smoothed to reduce the number of artefacts from the elevation maps (for example, an unrealistic flood elevation between two adjacent cells, i.e., wall of water).

For the first iteration, each source point will propagate to the next cell using either a diffusion or kinematic wave to compute the elevation at the empty neighbouring cells (subscript $\mathrm{C}$ ). Figure 1 shows the neighbouring convention used by this algorithm, in which $z$ is the DTM elevation from the reference datum. Each source point will loop through the 8 neighbouring cells.

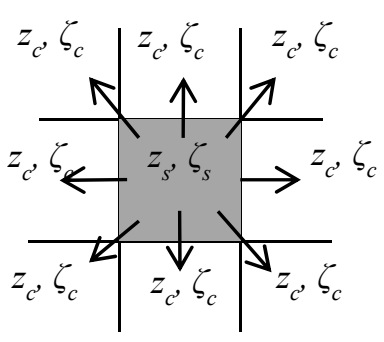

(a)

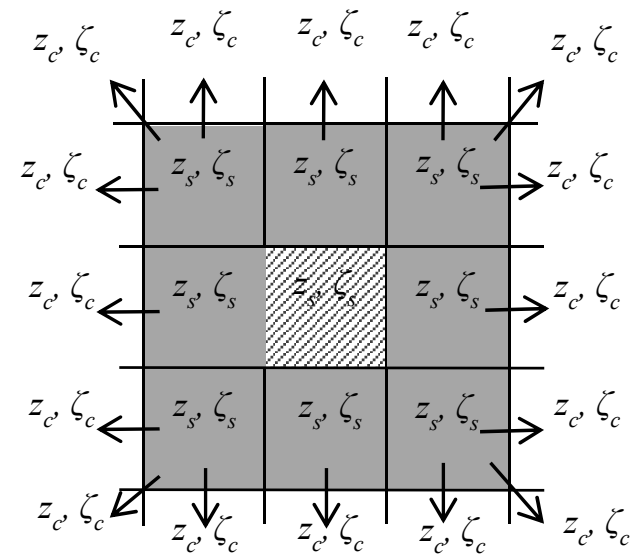

(b)

Figure 1: Neighbouring scheme and expansion steps for: a) 1st iteration and b) second iteration.

For each successive step, every neighbour cell at the previous step will become a source point and the original source point will be removed from the set of source points (Figure 1(b)). The iteration will continue until the ground elevation will not allow any further expansion from source cell; that is, when the list of source cells is empty, or when the maximum number of iterations is reached. After each expansion step, flooded cells' elevations are added in a smoothing array, while the source cell evaluated directly from the cross section will not be added, in order to prevent any alteration of the $1 \mathrm{D}$ solution along the cross section.

Thus, for each expansion step, the water elevation of each cell $\zeta_{C}$ will be calculated according to eqn. (2):

$$
\zeta_{C}=\zeta_{S}-\Delta E
$$

where, $\Delta E$ is the head drop per expansion step.

Because the kinematic wave propagates its peak without dissipation, and rearranging the kinematic terms of eqn. (1) and combining it with eqn. (2), the 
maximum water depth propagation computed from cell to cell in steady state conditions will assume the form:

$$
\zeta_{C}=z_{C}+X_{L(T)} \cdot\left(\zeta_{S}-z_{S}\right)
$$

Note that the source water depth propagates without dispersion in eqn. (3).

In contrast, in the case of a diffusion wave, the wave propagates to its maximum depth while reducing its peak during wave propagation. The dissipation $\Delta E$ can be calculated by rearranging the diffusion wave in eqn. (1) and evaluating the friction slope through Manning equation:

$$
\min \left(\Delta E=\Delta L \cdot S_{0} \cdot\left[\frac{n_{L(T)} \cdot V_{L(T)} \cdot \psi_{L(T)}}{\left(\zeta_{S}-Z_{S}\right)^{\frac{2}{3}}}\right]^{2}, \Delta E_{\max }\right)
$$

Herein, $\Delta L=\Delta X \cdot \theta$ is the distance from the cell centres, $\Delta X$ is the cell size, and $\theta$ is a direction factor equal to $\theta=1$ for horizontal and vertical neighbours $(\theta=1.41$ for diagonal cells), $S_{0}=\left|\zeta_{s}-\zeta_{c}\right|$ is the DTM slope, $n_{L(T)}$ is the longitudinal (transverse) Manning coefficient, $V_{L(T)}$ is the longitudinal (transverse) flow velocity, and $\psi_{L(T)}$ is the downhill scale factor. Manning's $n$ coefficient and velocity are set constant and do not depend upon the orography of the area. Therefore, $\psi$ and $S_{0}$ are used to scale the flow velocity from flat areas to steep areas, and obtain different energy dissipation values for catchments with different slopes.

The maximum drop $\Delta E_{\max }$ is enforced to reduce the energy dissipation in presence of artefact that may present high slope values.

The selection between kinematic and diffusion wave is based upon the definition of longitudinal and transversal wave propagation. In case of longitudinal wave propagation, the diffusion wave assumes velocity and Manning coefficients that approximate the wave propagation along the river centre line. Conversely, the transverse wave propagation assumes lower Manning's $n$, velocity and thus energy dissipation, to simulate propagation normal to the river centre line.

For transversal wave propagation, in fact, the free surface slope cannot be approximated using the ground slope and, thus, the kinematic assumption would lead to high error in predicting the water surface [7,9] and cannot be used to evaluate the water elevation.

In this study, the distinction between longitudinal and transverse wave propagation is achieved through the location of the expansion cell $C$ originating from a source point lying on a cross section.

In order to differentiate between the two different wave propagation, it is necessary to first introduce the Euclidean polygons. These polygons are defined as the sets of point with a minimum Euclidean distance from the source cross section. The DTM space is, therefore divided into different Euclidean polygons, corresponding to each cross section.

Wave propagation of cells within the Euclidean polygon will be assumed to have transverse wave propagation, while cells outside the Euclidean polygon will 
be assumed to have longitudinal wave propagation, and thus, will have higher energy dissipation to smoothly fill the gap between cross sections.

When the expansion cell lies inside the Euclidean polygon, the algorithm selects the water elevation to be the maximum of the two elevations computed by the diffusion or by the kinematic wave. During the longitudinal wave propagation (thus, outside the Euclidean polygon), the water elevation is evaluated as the minimum yielded by the kinematic and diffusion equations. Figure 2 shows the results of the expansion algorithm from the $1^{\text {st }}$ iteration to the final map extent (Rhone River near Martigny). Figure 2(a) shows the first step of the expansion algorithm. Here, the green line represents the cross section automatically generated from the catchment and flow line characteristics (green lines), while the green dots represent the source point location.
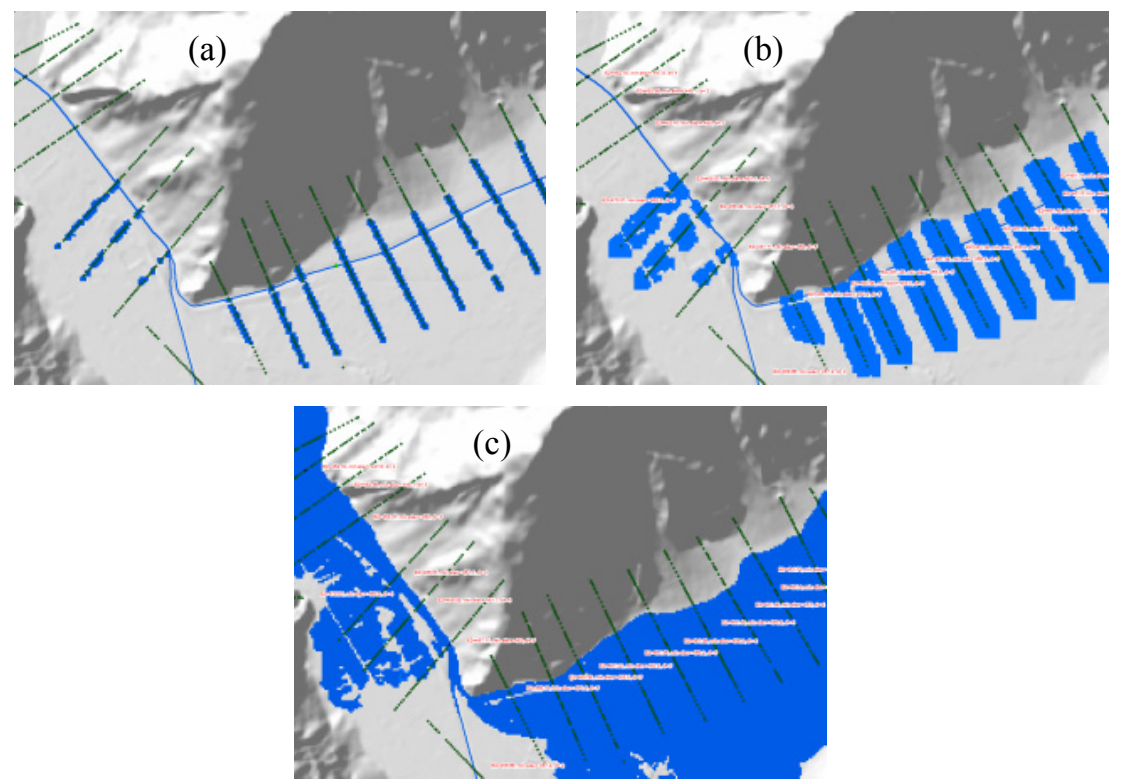

Figure 2: Example of modeled flood expansion (Rhone River near Martigny, $\mathrm{CH}$ ) at three different iterations: (a) 1st iteration, (b) 3rd iteration, (c) final extent.

Only the source points with an elevation above the DTM elevation can expand from the $1^{\text {st }}$ iteration, as shown by the flooded cells (blue) under the source point. Herein, not all the points have been activated to show the potential of the algorithm to flood area outside the cross section limit. As the algorithm executes, gaps between cross sections are filled in both directions (Figure 2(a)). Finally, as the algorithm proceeds, area outside the cross section extents will be flooded, in both longitudinal and transversal direction. As shown in the figure (see the lower right quadrant of Figure 2(c)), the flood extent map does not stop at the end of the cross 
section; rather, the algorithm is capable of expanding the modeled flood extent into those areas that would be left dry using traditional approaches.

\subsection{Levee breach and overtopping}

The general framework presented here, i.e., kinematic and diffusion wave propagation, can be used to evaluate the water depth from levee overtopping or breach to provide a solution that adapts to the morphology of a floodplain. The simplified 2D mapping algorithm has parameters selected to maximize the correspondence between the 1D solution and the 2D solution. This is done to prevent "wall of water" and map discontinuities at junctions observed using TINbased flood maps in which cross sections are not long enough to cover the extent of the floodplain. However, in cases of levee breach or overtop, the longitudinal dissipation may be either too low or too high; therefore, models of levee failure or overtopping need to be driven by different physical assumptions, volume carried by the hydrograph and wave duration.

Several reduced complexity models, or simplified models, have been developed to assess the risk of levee failure, overtopping, or inundation of urban areas. Typical approaches include models based on the discretisation of the diffusive wave equation on Cartesian grids [16], regular and irregular storage cell models [3, 5, 10-14] and raster based inertial models [15].

These reduced complexity methodologies are based on different assumptions, but they all aim to decrease the computational cost of assessing levee failure compared to fully physical $2 \mathrm{~d}$ shallow water solutions. However, these reduced complexity methodologies also analyze the evolution of the flood extent within a certain event, and, thus may need data post processing, which requires additional analysis and computational overhead, especially over a large domain.

In this paper, we use these types of risk maps to assess the maximum flood elevation and extents associated with a certain return period in case of levee breach and overtopping. Velocity within the floodplain will be neglected, due to the relatively lower velocity in the floodplain compared to the main channel.

Starting from the source points at the end of a cross section, the average depth can be evaluated using the information from the volume of water available within a certain time or from a given hydrograph.

To account for the added water volume that may come from a breach, the expansion parameters must be dynamically changed to adapt the solution to the average available volume associated with a certain return period.

For each step, the available volume is redistributed in the expanded area such that the average water depth for the next expansion multiplied by the total expanded area matches the available volume.

Thus, the expansion volume at the step $n, V^{n}$, is calculated as:

$$
V^{n}=A^{n} \bar{d}^{n}
$$

where: 


$$
\overline{\boldsymbol{d}}^{n}=\frac{\Delta V^{n}}{\Delta A^{n}}
$$

in which $\bar{d}^{n}=$ average depth at iteration $n, \Delta V^{n}=$ difference of volume expanded between step $n$ and $n-1, \Delta A^{n}=$ difference of extents between step $n$ and $n$-1, $A^{n}$ is total the area flooded at the step $n$.

The downhill scale parameter $\psi$ is then adjusted according to the normalized volume error:

$$
E^{n}=\left(V^{n}-V_{\max }^{n}\right) / A^{n}
$$

where, similarly to the method adopted by Liu and Pender (2010) [5], $V_{\max }{ }^{n}$ is the total maximum volume available from the breach hydrograph at the expansion step $n$ from the beginning of the simulation, either obtained from a historical event or from a simulated flood event. In this case, if the error has a positive bias, $\psi$ increases by a certain amount, in order to increase the dissipation for the next step and adjust the volume. Since each expansion step depends on the model resolution and not on a time step, the total volume available at each expansion step needs to be evaluated from the hydrograph associating a certain time step to each expansion step. In first approximation, this time step can be associated with typical flood plain velocity, although more complex formulation can assume a dependency on the average velocity evaluated from the flow surface gradient. Herein, the floodplain velocity $u_{\exp }$ will be assumed constant and needs to be calibrated to produce reasonable extents, as discussed later in the validation.

To be more explicit, for a triangular flood hydrograph of which the volume overtopping or flowing through the flood defence $V_{\max }$ is known, the volume at the expansion step $n$ will be calculated as:

$$
\begin{gathered}
V_{\text {max }}{ }^{n}=V_{\text {max }}\left(\frac{n}{n T P}\right)^{2} \frac{n T P}{n T E} \text { for } n<\mathrm{nTP} \\
V_{\text {max }}{ }^{n}=V_{\max }\left[1-\left(\frac{n T E-n}{n T E-n T P}\right)^{2}\right] \frac{n T P-n T E}{n T E} \text { for } n>\mathrm{nTP}
\end{gathered}
$$

where $n T P=T P /\left(\Delta x \cdot u_{\text {exp }}\right), n T P$ is the number of expansion iterations at the hydrograph peak TP, $n T E=T E /\left(\Delta x \cdot u_{\exp }\right)$ is the number of expansion iterations at the end of the hydrograph, $u_{\exp }=$ is the average flood expansion velocity, and $\Delta x$ is the cell grid size.

\section{Validation and discussion}

Modeled flood extents are validated by comparing the Ohio River Federal Emergency Management Agency (FEMA) 100-year flood maps to the modeled flood extent for the city of Cincinnati (Ohio), and by comparing gage station observations and modeled flood extents for the New Madrid 2011 breach [6].

The error between modeled flood elevation and observed flood gage elevation has been measured in terms of root mean square error (RMSE) between predicted and measured maximum elevation. The fit between two maps can also be 
expressed in terms of fraction of the inundation domain correctly assessed by the model [17]:

$$
F=\frac{\sum P\left(D_{1}, M_{1}\right)}{\sum P\left(D_{1}, M_{1}\right)+\sum P\left(D_{0}, M_{1}\right)+\sum P\left(D_{1}, M_{0}\right)}
$$

where the $P$ is the generic cell in both model $(M)$ and pilot domain $(D)$ either flooded (subscript 1) or dry (subscript 0 ). Modeled flood extents have been created using the U.S. national elevation dataset (NED) with a resolution of $30 \mathrm{~m}$.

Figure 3 compares the FEMA flood extent maps and the modeled flood extent (AE zones in light blue and $\mathrm{X}$ zones protected by levee in yellow) for the Greater Cincinnati region; the modeled flood extents were determined using the procedure described in this paper, in which water elevations are propagated from cross sections.

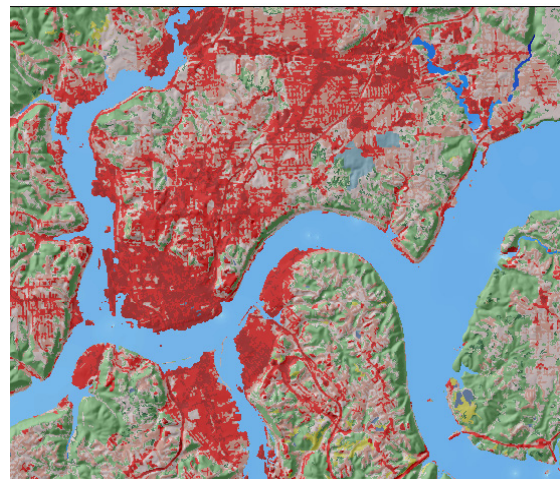

(a)

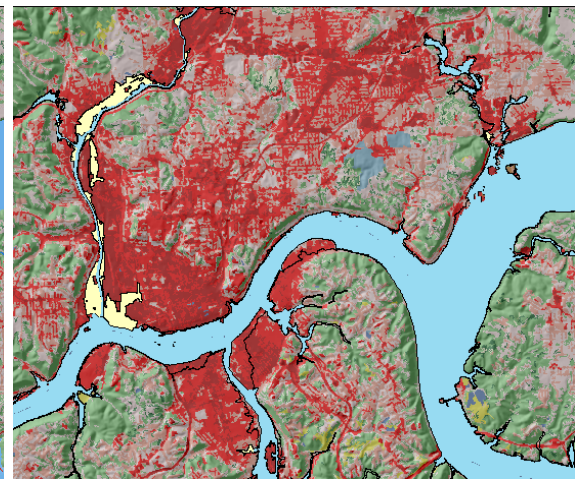

(b)

Figure 3: $\quad$ 100-year flood extent validation (Cincinnati, OH): (a) modeled flood extent, and (b) FEMA 100-year AE zone (light blue) and X protected zone (yellow). NOTE: red and pink colours represent the urban area extracted from the NLCD 2006 land use land cover data.

The agreement between the FEMA extent map and the modeled map in Cincinnati urban area considering both $\mathrm{AE}$ and $\mathrm{X}$ protected zones is $F=0.76$. The levee breach model has been validated by comparing the 2011 breach of Cairo, Illinois and the USACE simulation [6]. The Birds Point, Illinois, breach reached its maximum volume after 3 days of operation with a maximum volume of around $1.3 \cdot 10^{9} \mathrm{~m}^{3}$ breach. For reference, USACE provides a model of the 2011 event Cairo breach showing the floodway evolution after 120 hours (5 days) [6], which shows an average propagation velocity of $0.2 \mathrm{~m} / \mathrm{s}$. The USGS provides a large dataset of field measurements for this event (around 20 gage stations inside the floodway, shown by the green dots in Figure 4). The maximum value of each gage has been selected and used to calibrate the model. A range of different $u_{e x p}$ in the range $0.01 \mathrm{~m} / \mathrm{s}<u_{\text {exp }}<0.5 \mathrm{~m} / \mathrm{s}$ has been selected. According to these data, the minimum RMSE has been obtained using for $u_{e x p}=0.2 \mathrm{~m} / \mathrm{s}(\mathrm{RMSE}=1.21 \mathrm{~m}$; 
$F=0.7)$. Figure 4 compares the observed flood extent obtained from Landsat image to the modeled flood extent. Water extent from Landsat images have been extracted using the modified normalized difference water index (MNDWI [18, 19]), using a value of MNDWI $>0.3$ to detect water features on the image.

Two types of boundary conditions mainly affect the raster model, i.e., topography and Manning's n coefficient correlated with the land use. However, the results of both the general flood extent model and the levee failure model presented in this study fit well with observed flood data. Further, the performance of both models is comparable to that observed by Bates and De Roo [16] at similar DTM resolutions.

In addition, we noted that a simplified flood model that employs a DTM with coarser resolution produces modeled flood extent maps that fit less well to observed flood extents. For example, Bates and De Roo [16], observed that choosing a 25 meter resolution land use raster rather than $100 \mathrm{~m}$ resolution raster inflicts a $10 \%$ loss in fit quality between the modeled and observed flood extents." In both Ohio River and New Madrid floodplain, fine resolution DTM topography accounts for additional and localized levee protection, which cannot be correctly assessed using the original resolution and need to be manually surveyed and added on the DTM.

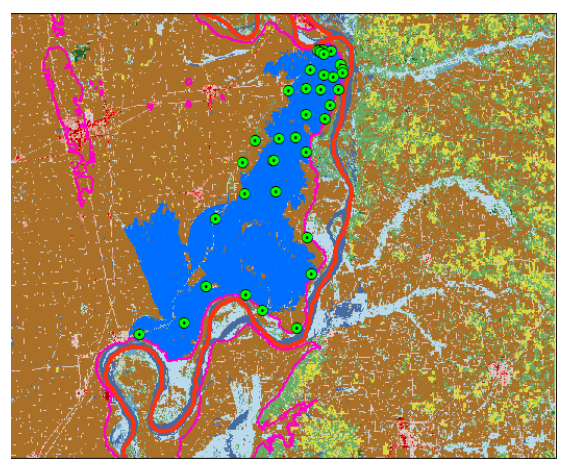

(a)

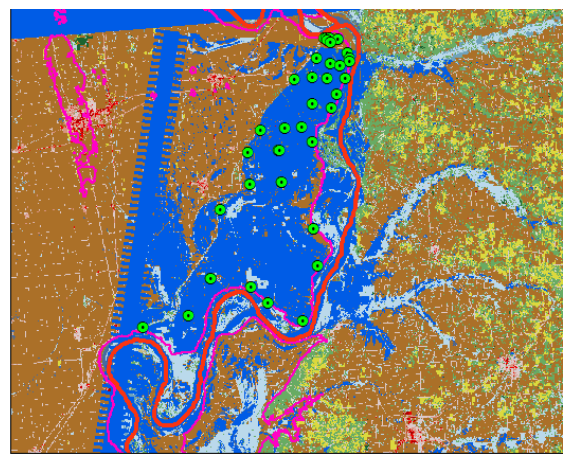

(b)

Figure 4: Comparison between (a) simulated levee failure with $u_{\text {exp }}=0.2 \mathrm{~m} / \mathrm{s}$ (green dots: USGS gage station; pink line: USACE accredited levees, red line: Mississippi river centre line) and (b) Landsat extracted water bodies (MNDWI $>0.3$ ) for New Madrid floodway.

Other biases may derive from the use of a global land use in both modelling approaches that may account for localized differences between predicted extents and satellite images. Moreover, the use of a single $u_{\text {exp }}$ used for the levee breach approach to estimate the amount of volume available for each expansion step may lead to depth underestimates during the first development phase, where flow expansion is generally faster due to a steeper free surface. In contrast, depth overestimates can occur during the last ponding expansion phase of the breach, 
where velocity may reach lowest expansion value. Finally, the model does not account for ponding and backwater effects, which further reduce the computational performance of this methodology.

\section{Conclusion}

A new framework to map 1D model results both along river flood plain and due to overtopping or levee failure has been presented. The model is based on simple development of kinematic and dynamic wave propagation. The modeled results are comparable to those produced by other simplified models, while maintaining a reduced computational cost. Boundary conditions, such as topography and land use, account for the majority of discrepancy between the FEMA flood map (where floodplains are manually surveyed) or historical inundation maps. Finally, further analysis of the sensitivity of the model on the parameters used to reproduce flood extent maps in both the general and levee framework should be conducted.

\section{References}

[1] Mapping the Zone: Improving Flood Map Accuracy; National Research Council; Washington, DC: The National Academies Press, 2009.

[2] Horrit, M.S. \& Bates, P.D., Evaluation of 1D and 2D numerical models for predicting river flood inundation, Journal of Hydrology, 268, pp. 87-99, 2002.

[3] Krupka, M., Pender, G., Wallis, S., Sayers, P.B. \& Mulet-Marti, J., A rapid flood inundation model, In Proceedings of the 32nd Congress of the International Association For Hydraulic Research, pp 1-28, 2007.

[4] Büchele, B., Kreibich, H., Kron, A., Thieken, A., Ihringer, J., Oberle, P., Merz, B. \& Nestmann, F. Flood-risk mapping: contributions towards an enhanced assessment of extreme events and associated risks, Nat. Hazards Earth Syst. Sci., 6, pp. 485-503, 2006.

[5] Liu, Y. \& Pender, G., A new rapid flood inundation model, In Proceedings of the First IAHR European Congress, ed. S. Arthur, Edinburgh, UK, 2010.

[6] DeHaan, H., Stamper, J. \& Walters, W., Mississippi River and Tributaries System 2011 Post-Flood Report, USACE, Mississippi Valley Division, 2012.

[7] Ponce, V.M., Li, R.M. \& Simons, D.B., Applicability of kinematic and diffusion-models, Journal of the Hydraulics Division - ASCE, 104 (3), pp. 353-360, 1978.

[8] Mujumdar, P.P., Flood wave propagation - The Saint Venant Equation, Resonance, 6 (5), pp. 66-73, 2001.

[9] Singh, V.P. \& Aravamuthan, V., Errors of kinematic-wave and diffusionwave approximations for steady-state overland flows, Catena, 27 (3-4), pp. 209-227, 1996.

[10] Cunge, J.A.., Two-dimensional modeling of flood plains. Water Resources Publications, 17, 705-762, 1975. 
[11] Moussa, R. \& Bocquillon, C. On the use of the diffusive wave for modelling extreme flood events with overbank flow in the floodplain, Journal of Hydrology, 374, pp. 116-135, 2009.

[12] Castellarin, A., Domeneghetti, A. \& Brath, A., Identifying robust large-scale flood risk mitigation strategies: A quasi-2D hydraulic model as a tool for the Po River, Physics and Chemistry of the Earth, 36, pp. 299-308, 2011.

[13] Gouldby, B., Sayers, P., Mulet-Marti, J., Hassan, M. \& Benwell, D., A methodology for regional-scale flood risk assessment. Proceedings of the Institution of Civil Engineers - Water Management, 161, pp. 169-182, 2008.

[14] Falter, D., Vorogushyn, S., Lhomme, J., Apel, H., Gouldby, B. \& Merz, B., Hydraulic model evaluation for large-scale flood risk assessments, Hydrol. Process., 27, pp. 1331-1340, 2013.

[15] Bates, P.D., Horritt, M.S. \& Fewtrell, T.J., A simple inertial formulation of the shallow water equations for efficient two-dimensional flood inundation modelling, Journal of Hydrology, 387, pp. 33-45, 2010.

[16] Bates, P.D. \& De Roo, A.P.J., A simple raster-based model for flood inundation simulation, Journal of Hydrology, 236, pp. 54-77, 2000.

[17] Aronica, G., Bates, P. D. \& Horritt, M. S., Assessing the uncertainty in distributed model predictions using observed binary pattern information within GLUE, Hydrol. Process., 16, pp. 2001-2016, 2002.

[18] Xu, H., Modification of normalised difference water index (NDWI) to enhance open water features in remotely sensed imagery, International Journal of Remote sensing, 27 (14), pp. 3025-3033, 2006.

[19] Ho L.T.K., Umitsu M. \& Yamaguchi Y., Flood hazard mapping by satellite images and SRTM DEM in the Vu Gia - Thu Bon alluvial plain, Central Vietnam, International Archives of the Photogrammetry, Remote Sensing and Spatial Information Science, 38 (8), pp. 275-280, 2010. 